\title{
On Two Conjectures of Franz Hering About Convex Surfaces
}

\section{Tudor Zamfirescu}

Department of Mathematics, University of Dortmund, Postfach 500500, 46 Dortmund 50, Germany

\begin{abstract}
In this paper we show that from the point of view of the Baire categories for most convex bodies no shadow boundary is included in a hyperplane. A related, more quantitative question is also considered. It receives in general a negative answer and in the $C^{2}$-case a positive one, but remains open in the $C^{1}$-case.
\end{abstract}

\section{Introduction}

At the Oberwolfach Conference on Convex Bodies in 1986, Franz Hering formulated the following conjectures:

Let $K \subset \mathbb{R}^{d}$ be a $d$-dimensional convex body. For $x \notin K$, let $\partial(K, x)$ be the shadow boundary of $K$ with respect to $x$, i.e.,

$$
\partial(K, x)=\{y \in K: \operatorname{aff}\{x, y\} \cap \text { int } K=\varnothing\}
$$

aff $X$ denoting the affine hull of $X \subset \mathbb{R}^{d}$.

Conjecture 1. There are convex bodies $K$ such that $\operatorname{dim}$ aff $\partial(K, x)=d$ for all $x \notin K$.

Conjecture 2. For all convex bodies $K$ and any $\varepsilon>0$ there are an $x \notin K$ and $a$ hyperplane $H$ such that

$$
\max \{\Delta(y, H): y \in \partial(K, x)\}<\varepsilon \Delta(x, H),
$$

where $\Delta(z, Z)$ means the distance from $z \in \mathbb{R}^{d}$ to the set $Z \subset \mathbb{R}^{d}$.

It is clear that the second conjecture becomes more interesting if we do not allow $x$ to go far from $K$. So let us require that $\Delta(x, K) \leq 1$. From now on we always consider Conjecture 2 with this additional condition. 
We shall establish here the first conjecture and prove the second for a certain class of convex bodies, including those with a boundary of class $C^{2}$. In general, Conjecture 2 is not true, as I. Bárány recently discovered.

A topological space in which every open set is of the second (Baire) category is called a Baire space. We say that most elements of a Baire space have a certain property if all those enjoying it form a residual set, i.e., the complement of a set of the first category. Such elements are also called typical. It is well known that the space $\mathscr{K}$ of all $d$-dimensional convex bodies of $\mathbb{R}^{d}$ equipped with the Hausdorff metric is a Baire space. (For a survey on properties of typical convex bodies see [4] or [9].) For example, it is known that every convex surface has almost everywhere a finite curvature in any tangent direction [1]. This curvature may even vanish almost everywhere in any tangent direction (see de Rham's example of a convex curve of this type in [3]). Moreover, according to [7], most convex bodies have such boundaries.

As usual, we think of the projective space $\mathbb{P}^{d}$ as $\mathbb{R}^{d}$ plus the hyperplane at infinity. Clearly, the definition of $\partial(K, x)$ can be extended to any $x \in \mathbb{P}^{d} \backslash K$.

We use the following notations: $N(x)$ for the line through $x$ and the point of $K \in \mathscr{K}$ closest to $x, p_{F} A$ for the orthogonal projection of the set $A$ on the flat $F, m_{k}$ for the $k$-dimensional Hausdorff measure, and $D(A, B)$ for $\inf \{\|a-b\|: a \in A, b \in B\}$, where $A, B \subset \mathbb{R}^{d}$.

\section{Proof of the First Conjecture}

We prove Conjecture 1 by using Baire's well-known theorem on categories.

Theorem 1. For most convex bodies $K \in \mathscr{K}$,

$$
\operatorname{dim} \operatorname{aff} \partial(K, x)=d
$$

for all $x \in \mathbb{P}^{d} \backslash K$.

Proof. It is probably known and easily seen that the function $s$ defined on $\mathbb{P}^{d} \times \mathscr{K}$ by

$$
s(x, K)=\partial(K, x)
$$

is upper semicontinuous in both variables, with respect to inclusion and Hausdorff metric. This means, for $K \in \mathscr{K}$ and $x \in \mathbb{R}^{d}$, that, for any $\varepsilon>0$, there exists a $\delta>0$ such that, for every $K^{\prime} \in \mathscr{K}$ at Hausdorff distance less than $\delta$ from $K$ and for every $x^{\prime} \in \mathbb{R}^{d}$ with $\left\|x-x^{\prime}\right\|<\delta$, each point $y \in \partial\left(K^{\prime}, x^{\prime}\right)$ satisfies $\Delta(y, \partial(K, x))<\varepsilon$; similarly for $K \in \mathscr{K}$ and $x \in \mathbb{P}^{d} \backslash \mathbb{R}^{d}$.

Let

$$
\mathscr{K}_{n}=\left\{K \in \mathscr{K}: \exists x \text { with } \Delta(x, K) \geq n^{-1} \text { and } \operatorname{dim} \text { aff } \partial(K, x)<d\right\}
$$

We are going to prove that $\mathscr{K}_{n}$ is nowhere dense in $\mathscr{K}$. Consider the open set $\mathscr{O} \subset \mathscr{K}$ and choose $B \in \mathcal{O}$ smooth and strictly convex. By routine arguments we 
see the following:

(i) Putting, for any $K \in \mathscr{K}$,

$$
\nu(K)=\min _{x}\left\{\Delta\left(x, p_{N(x)} \partial(K, x): \Delta(x, K) \geq n^{-1}\right\},\right.
$$

the function $\nu: \mathscr{K} \rightarrow \mathbb{R}$ is continuous and $\nu(B)>n^{-1}$.

(ii) Putting, for any $K \in \mathscr{K}$,

$$
\begin{gathered}
\mu(K)=\min _{x}\left\{m_{d \cdots 1}(K \cap H): H \text { is a hyperplane, } K \cap H \neq \varnothing,\right. \\
\left.H \perp N(x), \Delta(x, H)=\nu(K), \Delta(x, K)=n^{-1}\right\}
\end{gathered}
$$

the function $\mu: \mathscr{K} \rightarrow \mathbb{R}$ is continuous and $\mu(B)>0$.

Put $b=\left[d^{3} / 4\right]+d$ and let $V_{\varepsilon}(b)$ be the maximal $(d-1)$-dimensional Hausdorff measure of a $(d-1)$-dimensional complex with $b$ vertices, whose (onedimensional) edges have length at most $\varepsilon$ each. Since $V_{\varepsilon}(b) \rightarrow 0$ for $\varepsilon \rightarrow 0$, we can find a number $\varepsilon_{0}>0$ such that $V_{\varepsilon_{0}}(b)<\mu(B) / 2$.

Now choose the polytope $P \in \mathcal{O}$ close to $B$, such that:

(1) Its vertices are in general position, in particular, for any vertices $v_{1}, \ldots, v_{d+1}$, aff $\left\{v_{1}, \ldots, v_{d+1}\right\}=\mathbb{R}^{d}$ and, for any facets $F_{1}, \ldots, F_{d+1}$ without a common vertex, $\bigcap_{i=1}^{d+1}$ aff $F_{i}=\varnothing$.

(2) The diameters of its facets are smaller than $\varepsilon_{0}$.

(3) $\nu(P)>n^{-1}$.

(4) $\mu(P)>\mu(B) / 2$.

In order to prove that $\mathscr{K}_{n}$ is nowhere dense, we now intend to show that $P \notin \overline{\mathscr{K}}_{n}$. Suppose, on the contrary, there is a sequence of points $\left\{x_{i}\right\}_{i=1}^{\infty}$ and a sequence of convex bodies $\left\{K_{i}\right\}_{i=1}^{\infty}$ converging to $P$ with $\Delta\left(x_{i}, K_{i}\right) \geq n^{-1}$, such that dim aff $\partial\left(K_{i}, x_{i}\right)=d-1$ for all $i$. Then $\partial\left(K_{i}, x_{i}\right) \subset H_{i} \cap$ bd $K_{i}$ for some hyperplane $H_{i}$. Since $\nu(P)>n^{-1}$, we have, from some value of $i$ on, $\partial\left(K_{i}, x_{i}\right)=H_{i} \cap$ bd $K_{i}$.

We may assume (take a subsequence if necessary) that $\left\{x_{i}\right\}_{i=1}^{\infty}$ converges to some point $x_{0} \in \mathbb{P}^{d}$ and $\left\{H_{i} \cap K_{i}\right\}_{i=1}^{\infty}$ converges to a compact convex set $G$. Then $\left\{\partial\left(K_{i}, x_{i}\right)\right\}_{i=1}^{\infty}$ converges to the relative boundary $\Gamma$ of $G$. By the upper semicontinuity of $s, \Gamma \subset \partial\left(P, x_{0}\right)$ (see Fig. 1). Clearly, $\operatorname{dim}$ aff $\Gamma=d-1, \Gamma=\operatorname{aff} \Gamma \cap b d P$,

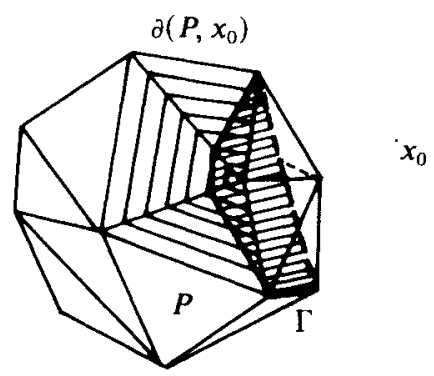

Fig. 1 
and $G$ is a $(d-1)$-dimensional polytope. Of course,

$$
\Delta\left(x_{0}, G\right) \geq \Delta\left(x_{0}, \operatorname{conv} \partial\left(P, x_{0}\right)\right) \geq \Delta\left(x_{0}, H_{0}\right),
$$

where $H_{0}$ is the hyperplane orthogonal to $N\left(x_{0}\right)$, which satisfies $H_{0} \cap P \neq \varnothing$ and $\Delta\left(x_{0}, H_{0}\right)=\nu(P)+\Delta\left(x_{0}, P\right)-n^{-1}$.

Since

$$
p_{H_{0}} G \supset P \cap H_{0},
$$

we have

$$
m_{d-1}(G) \geq m_{d-1}\left(p_{H_{0}} G\right) \geq m_{d-1}\left(P \cap H_{0}\right) \geq \mu(P)>\mu(B) / 2>V_{\varepsilon_{0}}(b) .
$$

Since every edge of $G$ lies in some facet of $P$ and therefore has length smaller than $\varepsilon_{0}, G$ has more than $b=\left[d^{3} / 4\right]+d$ vertices. Thus $\Gamma$ has more than $d^{3} / 4+d$ vertices, whereas $\partial(P, x)$ is the union of $\Gamma$ with at most $d$ facets $F_{1}, \ldots, F_{k}$ of $P$ (not more, because of the general position of the vertices of $P$ ). Since the intersection of a $(d-1)$-simplex with a hyperplane is a polytope with at most $d^{2} / 4$ vertices, $\Gamma$ has at most $k d^{2} / 4 \leq d^{3} / 4$ vertices in $\bigcup_{i=1}^{k} F_{i}$, which implies that more than $d$ vertices of $\Gamma$ are vertices of $P$ as well. But this contradicts their general position.

It follows that $P \notin \overline{\mathscr{K}}_{n}$. Thus, $\mathscr{K}_{n}$ is nowhere dense and $\bigcup_{n=1}^{\infty} \mathscr{K}_{n}$ is of the first category in $\mathscr{K}$, which proves the theorem.

Mani-Levitska [6] proved that - in the case of parallel "light rays"- there are classes of convex bodies $K$ which can cover all their shadows (i.e., there is for any $x \in \mathbb{R}^{d} \backslash\{0\}$ a rigid motion $c$ such that $\left.c\left(p_{x^{i}} K\right) \subset K\right)$, and classes of convex bodies which cannot cover all their shadows. He also observes (private communication) that every convex body which can cover all its shadows must have a planar shadow boundary. This together with Theorem 1 shows that most convex bodies cannot cover all their shadows. In fact more can be shown: those convex bodies which can cover all their shadows form a nowhere dense set.

\section{On the Second Conjecture}

With Bárány's kind permission, I reproduce here his example which shows that Conjecture 2 is false. In $\mathbb{R}^{3}$, let a plane $\Pi$ and a line $\Lambda$ be orthogonal and consider a circle $C \subset \Pi$, an ellipse $E \subset \Pi$ close to $C$, and a line segment $S \subset \Lambda$, all with midpoint in $\Pi \cap \Lambda$. Let $U$ be the union of all half-lines with the endpoint at one endpoint of $S$ and meeting conv $C$, and let $V$ be the union of all half-lines with the endpoint at the other endpoint of $S$ and meeting conv $E$. The convex body $K=U \cap V$ is a counterexample to Conjecture 2 .

We see that in the above construction, bd $K$ has a vanishing Gauss curvature at every smooth point, and any example based on Bárány's idea must have this property. It is natural to ask whether or not the conjecture does however hold for convex surfaces with a finite but not vanishing Gauss curvature at some point. In this section we give an answer to this question. 
In order to formulate Theorem 2, let us denote, for $K \in \mathscr{K}$, by $\rho_{i}^{\tau}(x)$ and $\rho_{s}^{\tau}(x)$ the lower and upper radii of curvature at $x \in \mathrm{bd} K$ in the tangent direction $\tau$ (for definitions see [2]). If $\rho_{i}^{\tau}(x)=\rho_{s}^{\tau}(x)$, let $\rho^{\tau}(x)$ denote the common value; then we say that bd $K$ has curvature $\left(\rho^{\tau}(x)\right)^{-1}$ at $x$ in direction $\tau$.

Let $N_{z}$ be the outer normal (with $z$ removed) at a smooth point $z$ of a given convex surface.

Theorem 2. If $K \in \mathscr{K}$ and bd $K$ has, for some smooth point $z$ and every tangent direction, a nonvanishing finite curvature, then, for $x \in N_{z}$, there is a hyperplane $H_{x}$ such that

$$
\frac{\max \left\{\Delta\left(y, H_{x}\right): y \in \partial(K, x)\right\}}{\Delta\left(x, H_{x}\right)} \rightarrow 0 \quad \text { as } \quad x \rightarrow z
$$

Proof. Let

$$
H_{x}=\{u:\langle u-2 z+x, x-z\rangle=0\}
$$

Also let $\tau$ be a tangent direction in $z$ and

$$
r<\rho^{\tau}(z)<r+\varepsilon
$$

Let $C_{1}$ be the half-circle of radius $r$ tangent to bd $K$ in $z$, lying in the half-plane $H$ bounded by the line $L$ through $x, z$, in direction $\tau$, and not disjoint from int $K$ (see Fig. 2). The line $T$ through $x$, tangent to $C_{1}$ in a point $t$, cuts the circle $C_{2}$ of radius $r+\varepsilon$, tangent to bd $K$ in $z$, coplanar with the preceding half-circle and

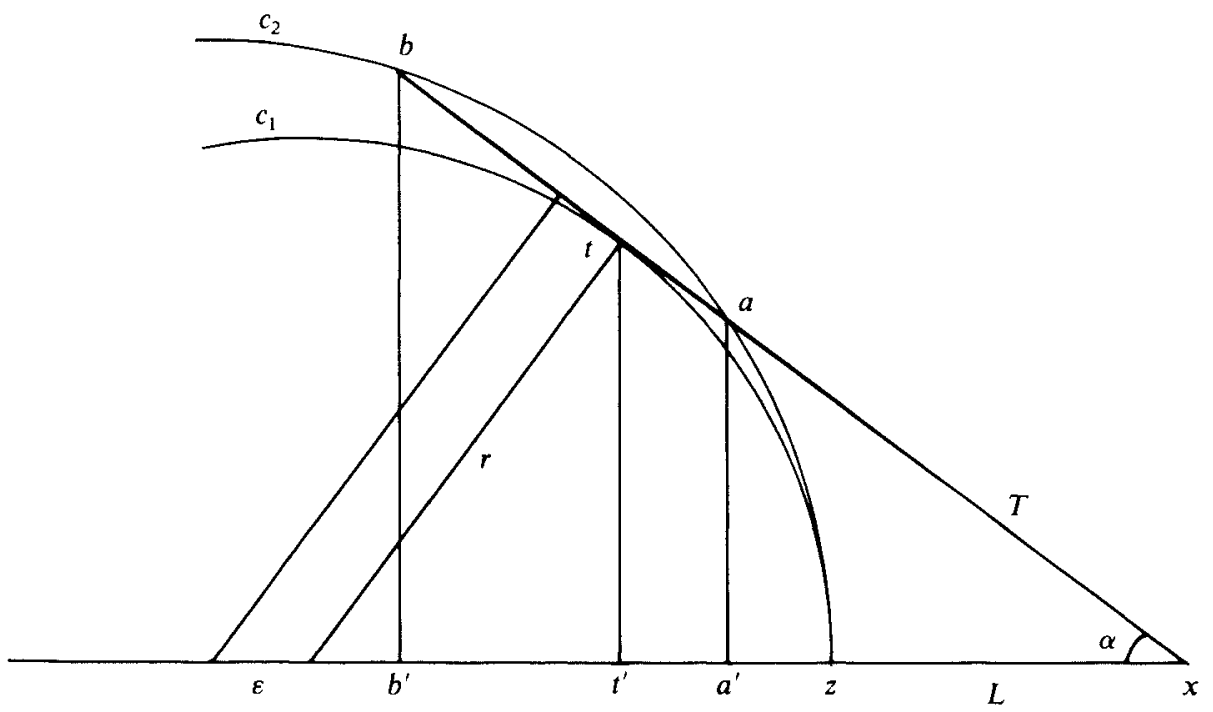

Fig. 2 
surrounding $t$, in two points $a, b$. Let $a^{\prime}, b^{\prime}, t^{\prime}$ be the orthogonal projections of the points $a, b, t$ on the line $L$. Clearly, any contact point $t_{\tau}$ of the line through $x$ tangent to bd $K \cap H$ has a point between $a^{\prime}$ and $b^{\prime}$ as projection on $L$. Let

$$
0<r_{1}<\min _{\tau} \rho^{\tau}(z) \leq \max _{\tau} \rho^{\tau}(z)<\infty
$$

and, for every tangent direction $\tau$, take $r>r_{1}$. The indicatrix at $z$ being convex [2], the above use of "min" and "max" is allowed and the extrema are neither 0 nor $\infty$.

If $\alpha$ is the angle between $T$ and $L$, then

$$
\|a-b\|=2 \sqrt{\varepsilon^{2} \cos ^{2} \alpha+2 r \varepsilon(1-\sin \alpha)}
$$

and

$$
\left\|t^{\prime}-x\right\|=r \cos ^{2} \alpha \sin ^{-1} \alpha
$$

whence

$$
\frac{\left\|a^{\prime}-b^{\prime}\right\|^{2}}{\left\|t^{\prime}-x\right\|^{2}}=\frac{\sin ^{2} \alpha}{r^{2}}\left(4 \varepsilon^{2}+8 r \varepsilon \frac{1-\sin \alpha}{\cos ^{2} \alpha}\right)<\frac{1}{r_{1}^{2}}\left(4 \varepsilon^{2}+8 r_{1} \varepsilon\right) .
$$

On the other hand,

$$
r^{2}=\left(r-\left\|t^{\prime}-z\right\|\right)(r+\|x-z\|)
$$

which yields

$$
\frac{\left\|t^{\prime}-z\right\|}{\left\|t^{\prime}-x\right\|}=\frac{1}{2+\|x-z\| / r}>\frac{1}{2+\|x-z\| / r_{1}}
$$

Now, if $x \rightarrow z$ and $\varepsilon \rightarrow 0$, then

$$
\frac{\left\|t^{\prime}-z\right\|}{\left\|t^{\prime}-x\right\|} \rightarrow \frac{1}{2} \text { and } \frac{\left\|a^{\prime}-b^{\prime}\right\|}{\left\|t^{\prime}-x\right\|} \rightarrow 0
$$

$\tau$-uniformly. Observing that

$$
\frac{\left\|t^{\prime}-z\right\|}{\|x-z\|} \rightarrow 1 \text { and }\|x-z\|=\Delta\left(z, H_{x}\right)
$$

we get

$$
\frac{\Delta\left(z, H_{x}\right)}{\left\|t^{\prime}-z\right\|} \rightarrow 1 \text { and } \frac{\Delta\left(x, H_{x}\right)}{\left\|t^{\prime}-x\right\|} \rightarrow 1
$$

whence

$$
\frac{\Delta\left(t^{\prime}, H_{x}\right)}{\Delta\left(x, H_{x}\right)} \rightarrow 0 \text { and } \quad \frac{\left\|a^{\prime}-b^{\prime}\right\|}{\Delta\left(x, H_{x}\right)} \rightarrow 0
$$


again $\tau$-uniformly. Finally, from

$$
\Delta\left(t_{\tau}, H_{x}\right) \leq\left\|a^{\prime}-b^{\prime}\right\|+\Delta\left(t^{\prime}, H_{x}\right)
$$

it follows

$$
\frac{\Delta\left(t_{\tau}, H_{x}\right)}{\Delta\left(x, H_{x}\right)} \rightarrow 0
$$

$\tau$-uniformly, which proves the theorem.

\section{Two Related Results}

The main idea of Bárány's counterexample is to have at every smooth point a vanishing curvature in one direction and a nonvanishing curvature in another direction. This does not happen in the case of a typical convex surface. But a typical convex surface does not satisfy the requirements of Theorem 2 either.

Theorems 3 and 4 below describe shadow-boundary properties which are weaker than that required in Conjecture 2 , but shared by most convex surfaces in $\mathbb{R}^{d}$.

Theorem 3. Let $K \in \mathscr{K}$ possess a smooth point $z \in \mathrm{bd} K$ and a tangent direction $\tau$ in $z$, such that bd $K$ does not have a positive curvature at $z$ in direction $\tau$. Then, for every $\varepsilon>0$, there is a point $x \in N_{z}$ such that

$$
D(H(x), \partial(K, x))<\|x-z\|<\varepsilon,
$$

where $H(x)=\{u:\langle u-z, x-z\rangle=0\}$

Proof. Let $T_{\xi}(\Xi)$ denote the tangent at $\xi$ to the curve $\Xi$. Consider the half-circle $C$ tangent to bd $K$ in $z$, lying in the half-plane $H$ bounded by the line $L \supset N_{z}$, in direction $\tau$, and not disjoint from int $K$. For $u \in C$, let $\left\{x_{u}\right\}=T_{u}(C) \cap N_{z}$ and, for $u \in$ bd $K \cap H$, let $\left\{y_{u}\right\}=T_{u}($ bd $K \cap H) \cap N_{z}$.

Case I. bd $K$ has no curvature at $z$ in direction $\tau$. In this case we choose the radius of $C$ to lie between $\rho_{i}^{\tau}(z)$ and $\rho_{s}^{\tau}(z)$. Also, let $v \in$ bd $K \cap H$ be such that $\left\|y_{v}-z\right\|<\varepsilon$. Let $A$ be one of the components of $K \cap C$ lying entirely between $v$ and $z$. Let $a$ be the endpoint of $A$ closer to $z$. Put $\{y\}=T_{a}(C) \cap H(x)$. Then

$$
\|a-y\|=\|y-z\|<\left\|y-x_{a}\right\|
$$

whence

$$
\Delta\left(a, H\left(x_{a}\right)\right)<\left\|x_{a}-z\right\| \leq\left\|y_{a}-z\right\| \leq\left\|y_{v}-z\right\|<\varepsilon .
$$

Hence

$$
D\left(H\left(y_{a}\right), \partial\left(K, y_{a}\right)\right)<\left\|y_{a}-z\right\|<\varepsilon .
$$


Case II. bd $K$ has vanishing curvature at $z$ in direction $\tau$. If the radius of $C$ is large enough, $C \cap$ bd $K$ consists of $z$ and another point $w$. Let the radius of $C$ tend to infinity. Then $w$ converges to $z$. Choose $C$ such that $\left\|y_{w}-z\right\|<\varepsilon$. As before,

$$
\Delta\left(w, H\left(x_{w}\right)\right)<\left\|y_{w}-z\right\|<\varepsilon,
$$

whence

$$
D\left(H\left(y_{w}\right), \partial\left(K, y_{w}\right)\right)<\left\|y_{w}-z\right\|<\varepsilon .
$$

The theorem is completely proved.

Corollary. For most $K \in \mathscr{K}, \mathrm{bd} K$ is smooth [5] and for most and almost all points $z \in$ bd $K$ and for any $\varepsilon>0$, there are points $x \in N_{z}$ such that

$$
D(H(x), \partial(K, x))<\|x-z\|<\varepsilon .
$$

For a proof it suffices to combine Theorem 3 above with Theorem 2 in [7] and Theorem 2 in [8].

Theorem 4. For most $K \in \mathscr{K}$, bd $K$ is smooth [5] and for almost all points $z \in$ bd $K$ and any $\varepsilon>0$, there are points $x \in N_{z}$ such that $\|x-z\| \leq 1$ and

$$
\frac{D(H(x), \partial(K, x))}{\|x-z\|}<\varepsilon .
$$

Proof. Let $a>0$. Consider two convex functions $f, g:[-a, a] \rightarrow[0, \infty)$ differentiable at 0 , satisfying $f(0)=f(0)=0$ and $g(x)=g(-x)$ for all $x \in(0, a]$.

In [10], we said that the graph of $f$ has a $g$-contact at $(0,0)$ if there is no neighborhood of 0 on the $x$-axis where $f(x) \geq g(x)$. It is easily seen that all results in [10] remain true if we replace, in the definition of a $g$-contact, the neighborhoods of 0 by intervals having 0 as an endpoint. We use here this stronger notion of a $g$-contact and a result from [10].

We can find a differentiable function $g$ such that if $(\beta(x), 0)$ is the intersection of the tangent at $(x, g(x))$ to the graph of $g$ with the $y$-axis and $\gamma(g, x)$ denotes $g(x)|\beta(x)|^{-1}$, then $\lim _{x \rightarrow 0} \gamma(g, x)=0$.

Let, for example,

$$
g(x)= \begin{cases}\mathrm{e}^{-|x|^{-1}} & \text { for } x \in\left[-\frac{1}{2}, 0\right) \cup\left(0, \frac{1}{2}\right], \\ 0 & \text { for } x=0 .\end{cases}
$$

For this convex function $g$, we have

$$
\beta(x)=g(x)-x g^{\prime}(x)=e^{-x^{-1}}\left(1-x^{-1}\right),
$$

whence, indeed,

$$
\lim _{x \rightarrow 0} \frac{g(x)}{|\beta(x)|}=\lim _{x \rightarrow 0}\left(x^{-1}-1\right)^{-1}=0
$$


Consider, for $k \in \mathbb{N}$, the function $g_{k}:\left[-\frac{1}{2}, 0\right) \cup\left(0, \frac{1}{2}\right]$ defined by $g_{k}(x)=k g(x / k)$, with graph $G_{k}$. It is easily seen that $\gamma(g, x)=\gamma\left(g_{k}, k x\right)$.

Assume the graph $F$ of $f$ has a $g_{k}$-contact at $(0,0)$ for any $k \in \mathbb{N}$. We shall prove that, under this assumption, for any $\varepsilon>0$, there is some $x_{3} \in\left(0, \frac{1}{2}\right)$ such that $\gamma\left(f, x_{3}\right)<\varepsilon$. Indeed, since $\lim _{x \rightarrow 0} \gamma\left(g_{1}, x\right)=0$, we can find $\nu \in\left(0, \frac{1}{4}\right)$ such that $\gamma\left(g_{1}, x\right)<\varepsilon$ whenever $x \in(0, \nu)$. Because $F$ has a $g_{1}$-contact at $(0,0)$, there is some point $x_{0}<\nu$ such that $f\left(x_{0}\right)<g_{1}\left(x_{0}\right)$. Consider the line $L$ through $(0,0)$ and $\left(x_{0}, f\left(x_{0}\right)\right)$. This line meets $G_{1}$ in a point $\left(x_{1}, g_{1}\left(x_{1}\right)\right)$ with $x_{1}<x$. Take $k \in \mathbb{N}$ such that $k x_{1} \in\left(x_{0}, \frac{1}{2}\right)$. This is possible, because $x_{0}, x_{1} \in\left(0, \frac{1}{4}\right)$. Thus $\left(k x_{1}, g_{k}\left(k x_{1}\right)\right)$ lies below $F$. Since $F$ has a $g_{k}$-contact at $(0,0)$, there exists $x_{2} \in\left(0, k x_{1}\right)$ such that $f\left(x_{2}\right)<g_{k}\left(x_{2}\right)$. Let

$$
x_{3}=\max \left\{x \in\left(x_{2}, k x_{1}\right): f(x)=g_{k}(x)\right\}
$$

Clearly, $\gamma\left(f, x_{3}\right) \leq \gamma\left(g_{k}, x_{3}\right)$. On the other hand, $\gamma\left(g_{k}, x_{3}\right)=\gamma\left(g, x_{3} / k\right)$. Since $x_{3}<k x_{1}$, we have $x_{3} / k<\nu$, hence $\gamma\left(g, x_{3} / k\right)<\varepsilon$. It follows that $\gamma\left(f, x_{3}\right)<\varepsilon$, too.

By Theorem 10 in [10], on most corvex surfaces, at almost every point, all normal sections have a $g_{k}$-contact at that point. With this remark the proof finishes.

We end the paper with the following open questions.

Problem 1. Is Conjecture 2 true for a smooth convex body?

Problem 2. Is Conjecture 2 true for a typical convex body?

\section{Acknowledgement}

Thanks are due to the referees for their useful comments.

\section{References}

1. A. D. Aleksandrov, Almost everywhere existence of the second differential of a convex function and some properties of convex surfaces connected with it (Russian), Vahenye Zapiski Leningrad. Gos. Univ. Mat. Ser. 6 (1939), 3-35.

2. H. Busemann, Convex Surfaces, Interscience, New York, 1958.

3. G. de Rham, Sur quelques courbes définies par des équations fonctionelles, Rend. Sem. Mat. Univ. Politec. Torino, 16 (1956/57), 101-113.

4. P. M. Gruber, Results of Baire category type in convexity, in: Discrete Geometry and Convexity, 163-169, Annals of the New York Academy of Science, Vol. 440, 1985.

5. V. Klee, Some new results on smoothness and rotundity in normed linear spaces, Math. Ann. 139 (1959), 51-63.

6. P. Mani-Levitska, Can you cover your shadows?, Discrete Comput. Geom. 1 (1986), 45-58.

7. T. Zamfirescu, The curvature of most convex surfaces vanishes almost everywhere, Math. $Z .174$ (1980), 135-139. 
8. T. Zamfirescu, Nonexistence of curvature in most points of most convex surfaces, Math. Ann. 252 (1980), 217-219.

9. T. Zamfirescu, Using Baire categories in geometry, Rend. Sem. Mat. Univ. Politec. Torino 43 (1985), 67-88.

10. T. Zamfirescu, Curvature properties of typical convex surfaces, Pacific J. Math. 131 (1988), 191-207.

Received May 2, 1988, and in revised form November 17, 1988. 